\section{Image Stitching Using Photoshop}

\author{
Jerry Sedgewick \\ University of Minnesota \\ sedge@umn.edu
}

Several images can be stitched together (sometimes referred to as "montaging") through the use of Photoshop. Edges of the images can be feathered and the process automated to reduce the hours of work that can go into this procedure.

Before going any further, let me be quick to say that more than one company has developed protocols to accomplish image stitching. When your lab is looking at large fields, while needing to resolve fine details, then I would strongly recommend the purchase of a system designed for this purpose. Issues surrounding motorized stage accuracy, problems with tilting when a camera is perched on a long optical tube, and image to image correction for contrast, color and uneven illumination can all contribute to confounding issues when image stitching. These companies have worked through these problems with off-the-shelf systems.

When $1 \mathrm{x}$ to $2 \mathrm{x}$ resolution is needed for brightfield illumination, microscope slides can be scanned on some slide scanners (the Nikon $35 \mathrm{~mm}$ film scanners, for example) and for fluorescent illumination, microarray scanners can be used.

For the once-in-a-blue-moon user, however, Photoshop works as an alternative. Before attempting image stitching, you may want to verify that it cannot easily be done in a program designed for that purpose (such as Panavue, or the image stitching protocol included with Photoshop Elements). Typically, any image series will work as long as no large areas of white or black exist, which is almost sure to happen with scientific images at the outer edges of the sample. In the end, these programs work well for photos of mountain ranges, but poorly for scientific images.

\section{Motorized stage and imaging system.}

You will need to locate a decent motorized stage that has been checked for $x$ and $y$ alignment against a microscope grid. In my own experience, motorized stage accuracy is commensurate with the cost, and it seems that costs need to be closer to $\$ 20,000$ than to $\$ 10,000$ for the accuracy needed for repeatable results. Calibration grids imprinted upon microscope slides can be obtained from your microscope salesman or from Microbrightfield, Inc. The $x$ and $y$ borders of these grids must move in parallel to the edge of your image area versus moving at an angle. Check that by finding the $x$ and $y$ border of the grid, and then moving the $x$ or $y$ motors with the joystick while observing parallelism. Sometimes a camera that is perched on a phototube is not parallel to the stage. A bubble level can be placed on a camera, assuming the back of the camera and the chip are parallel and this can be compared to what the bubble level reveals when placed on the stage. In this way, the direction of tilt can be determined and you can figure out how to correct the tilt (perhaps another good use for duct tape!).

I have had the best success with motorized stages when collecting across in the $x$ direction, having the stage move down to the next row, and then having the stage move across in the opposite direction, and so on. This creates some difficulties with file naming (the first row counts up, the second row counts down, and so on), but that can be solved by renaming later on, or by providing two actions (more on that later).

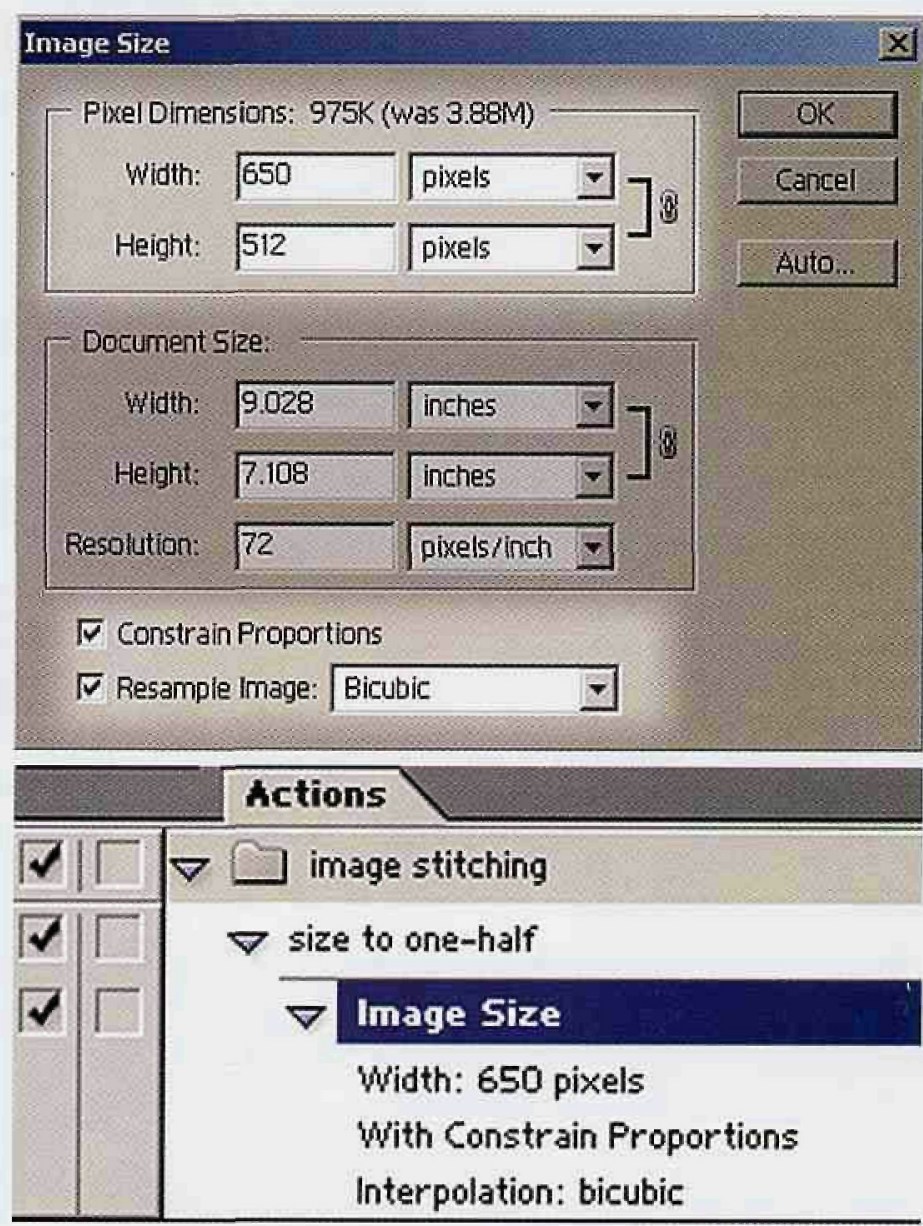

Fig2. (Top) For high resolution cameras, you may want to cut image sizes in half. Check first, however, to determine whether important information is lost and then decide upon the percentage of file size reduction. Unless the inage is being reproduced on a poster, the final image need be no larger than the size of a cover (typically, $8.5 \times 11$ inches at 300 or 400 pixels per inch: $3400 X 4400$ pixels at 400 ppi). (Middle) Note that the size reduction is done according to pixels as the unit of measurement, making the "Document Size" entries unimportant. Be sure Constrain Proportions and Resample innge are checked. Make an action while doing this so that this action an be applied to all your inages automatically. As a part of your action, you should not include opening the image (File $>$ Open). (Bottom) Use File $>$ Autonate $>$ Batch to accomplish automation. If you wish to apply the automation to all the file folders, then check Include All Subfolders. Otherwise, you can work folder by folder and save image files to new folders if you wish to preserve original images at high resolution. In that instance, click on the arrow next to Destination to reveal drop down list and make the destination Folder. 
New

Name: row1

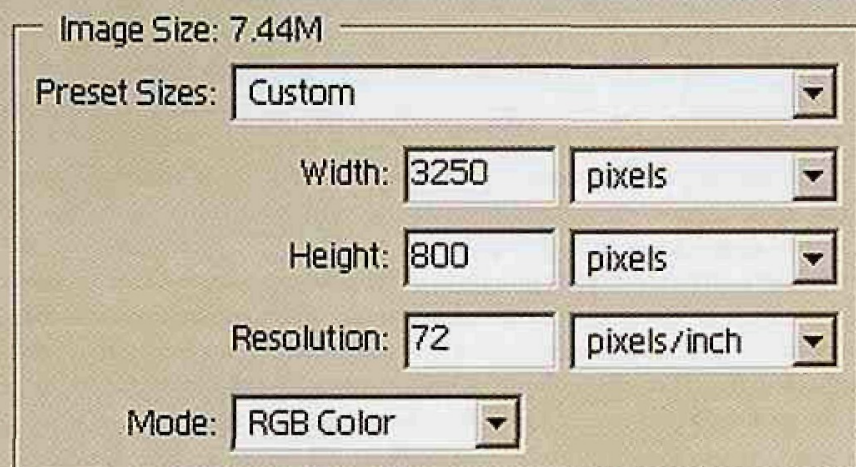

Batch

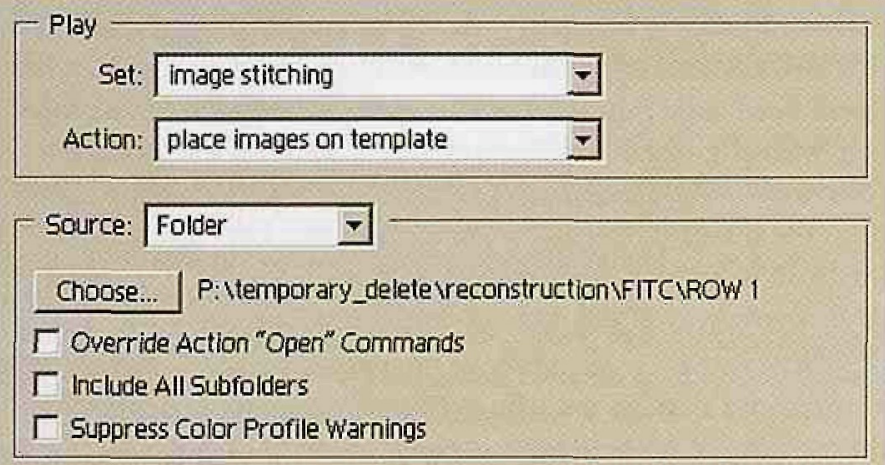

\section{Destination: None}

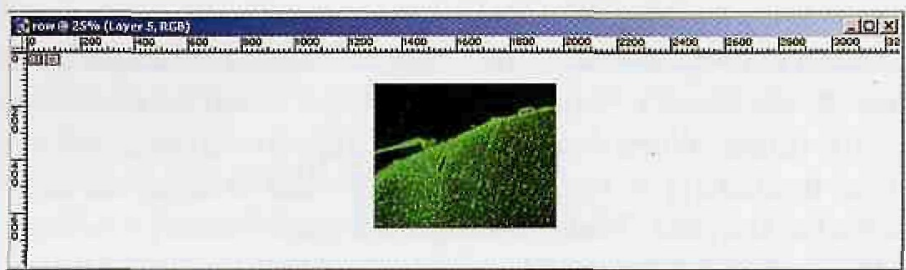

Fig3. (Top) Make a template into which a row of images can be placed; or, if you are working with a handful of image files, then make the template somewhat larger than the sum of the $\mathrm{x}$ and $\mathrm{y}$ dimensions of all images together. In this instance, the template is the sum of 5 image files (5 at 650 pixels for a sumt-or product-of 3250 pixels). Again, note that the Resolution does not need to be changed when working with pixels as the unit of measurement. You can record this as an action to be used for subsequent rows. Give yourself additional room in case the images form a "stairstep." (Middle) Make an action that includes Select All, Cut, Close Image, Paste (image of this action not shown). Then apply this acrion using File $>$ Automate $>$ Batch to the first row. (Bottom) All images should be stacked upon one another as layers. Remember that you should do all the above by starting with a screen cleared of all inage files, and do not zoom in on the template before automating the stacking of images (otherwise, the images will not be exactly centered, making the next steps more difficult to accomplish).

Uneven illumination across the field is almost always a given. If your imaging system provides shading correction (also called flatfield or blank field correction), then use this protocol when obtaining images. Otherwise, uneven illumination will have to be corrected later in Photoshop (not covered in this article). Especially with low magnification (using $2 \mathrm{x}, 4 \mathrm{x}$ or $10 \mathrm{x}$ objectives), issues with uneven illumination become near-impossible to overcome. You may want to overlap images to a larger degree (e.g., one-third image overlap) since most illumination problems occur at the left or right hand sides of the field.

Be sure to provide plenty of overlap from one row to another, especially critical when a less accurate motorized stage is used, or when uneven illumination is dramatic. The amount of time it takes to set up this method and to collect images results in greater frustration when the bottom images do not meet up with the tops. This may go without saying, but images should be named with 3- or 4-digit numbering extensions (e.g., "red001," "red002," etc.) to avoid problems with the order later on.

Finally, cameras MUST be set to a manual exposure. If this isn't done, then each image will be exposed slightly differently leading to brighter and dimmer images so that all do not match in terms of brightness. Find the ideal exposure on the brightest area of interest and let all other areas be exposed similarly. The brightest area may take a while to locate, but it is well worth the effort.

\section{Steps in Photoshop: Reduce File Sizes}

Once images are collected, the first matter to be resolved has to do with file sizes. With the advent of megapixel CCD's, file sizes can easily be over 4 megabytes for each color image (for TIFF files; avoid JPEG images, since these result in a loss of image information). All but the most robust computers can handle a great number of these files handily. Often, a decision about how far to reduce file sizes must be made. This can be done by experimenting with a single representative image to check the loss of important detail against varying cuts in the file sizes (what I mean here is the reduction of PIXELS across and down). I start by reducing the file to one-half the number of pixels across and down using Image Size under Image on the menu (be sure Resample is checked when doing so). Then I make a decision about whether to increase or reduce the file size further based upon whether important details remain.

If more than one camera exists in your lab, use the camera with the LEAST pixels across and down. That may seem counterproductive-after all, this process is being done to increase resolution - but the limiting factor, ultimately, will be the number of pixels across and down in the final, image-stitched photo: even full-size reproductions in scientific journals will only require 4400 pixels across in the long dimension. Not many of these images are destined for posters.

The idea of reducing file sizes makes all of us uneasy. For some reason it's more comforting to make the montage of images at full resolution, and then make that image smaller in file size than it is to throw away resolution at the get go. After one experience with even smaller montages of 12 or so images, users often change heart because a computer can take so long to plug away; or, worse, it crashes.

\section{Place Images into Folders by Rows or Columns}

Again, because of file size issues and the computer requirements for crunching through large numbers of images, it is best to place into folders either rows or columns, especially for larger numbers of images. Label the folders "1stRow001," "1stRow002," and so on. In Photoshop, the images will be stitched together into continuous rows or columns first, and then the completed rows or columns will be stitched from top to bottom or side to side in a second series of steps. 


\section{Steps in Photoshop.}

Under File $>$ New make a template upon which to paste your images. The template needs to be as long horizontally (assuming rows) as the number of images collected. Simply multiply out the pixels across in a single image by the number of photos in that row. Thus, if a single image is 1000 pixels across and you've downsampled it to 500 pixels, and you have 20 images across, you'd set the new image width to 10,000 pixels. Set the height to give enough room for slop in the stage. Thus, if the height of each image is 800 pixels, set the height to 1200 or so, just in case the stage had moved uphill or downhill, causing "staircasing"of the images. Excess space can be cropped off later.

The template will open so that it is visible from one end to the other. Do not zoom in! The next step requires that all images be pasted in the centermost position on the template, and that only happens when the entire image is visible on the screen.

\section{Make an Action.}

Open the Actions dialogue box (under Window $>$ Show Actions). Click on the upper right arrowhead and select New Set from the drop down list (for Photoshop $5 \mathrm{x}$ and above). Name this set "Image Stitching." Again reveal the drop down list and select New Action. Name it. This action will be used for subsequent rows in order to save time.

Copy first image onto the template while recording the action.

Click on the record button (the round, red button) to turn it off for the moment. Open the first image from the first row, then click the record button (this is done so that the action does not think it only needs to open that particular image file). Under Select choose $A l l$, under Edit choose Cut, under Image choose Close (don't save), under Edit choose Paste (to paste the cut image onto the template). Stop recording the action by clicking the Stop button (the black square icon) at the bottom left of the actions dialogue box.

\section{Automate pasting onto the template.}

You have now successfully created an action that you need to apply to every image in the folder you have named "Row 1." Now automate the process of opening each image in Row 1 so that all can be pasted onto the template. First you will need to eliminate the image you have already pasted onto the template so that the template can be clear of images. Click on the template, choose the Layers dialogue box (under Window > Show Layers), then drag the layer into the trash icon at the bottom of the Layers dialogue box, or click on the arrowhead at the top, right and choose Delete Layer from the drop down list.

Under File, choose Automate > Batch. In this dialogue box, you will need to choose the Source for the first row (the folder named "Row $\left.1^{\prime \prime}\right)$. Click Okay, and all the images in that folder will be placed on the template, one above the other (see Figure 3).

Create a Second Action, move images into place layer by layer.

Again reveal the drop down list in the Actions dialogue box and choose New Action. I name this

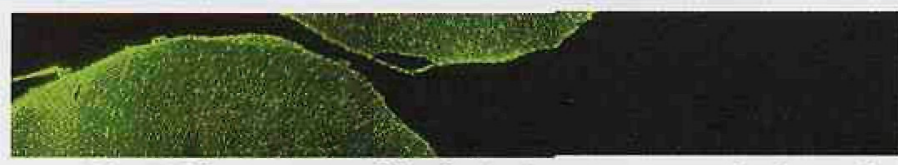

Fig4. While you are carefully aligning images next to each other, and while zooming in and out to be sure you have matched up features, be sure to record what you've done as an action. Start with layer 1, then 2 and so on. This action can then be applied to subsequent rows using Batch. Optional: You can determine $\mathrm{x}$ and $\mathrm{y}$ locations of your images by keeping the Info box open. In that manner you can figure the numeric positions of images as you move them into place. In Photoshop $5 x$, these volues can be typed in using the Numeric function (Edit $>$ Transform $>$ Numeric). In $6 x$, $7 x$ and $8 x$, the values can be typed in appropriate places at the bar under the menu. Use only the Transform function and then the boxes under the menu will appear. Note that some stages move at a different distance for the second image than for subsequent images along rows.

action "Stitch Into Row". You will need to start by making an adjustment layer so that contrast and brightness can be adjusted in order to see details. Because the first row is mostly black (for darkfield images) or mostly white (for brightfield), given that the first row records image areas above or below the area of interest, it will need to be modified so that every little speck can be seen to help with alignment of images. Under Layers on the menu, choose New Adjustment Layer, then Levels (or Brightness/Contrast). Zoom in to the image (control/command key $\&+$ key) and adjust contrast and brightness until every last speck is visible. This layer will be thrown away at the end, so don't hesitate to be bold.

Now you are ready to move images into place. Click on Layer 1 , and move that layer to the extreme left or right (depending on the movement of the stage and the placement of your microscope slide when acquiring images). Next click on Layer 2 and move that image so that fiduciary marks (specks) line up. Spend as much time as you need in order to line these up, zoom in and out, if necessary. The placement will not be recorded in the action until you choose Layer 3. Do the same for Layer 3, and for each layer until finished. When done, remove the Adjustment Layer (the top layer) by clicking on the top, right arrowhead to reveal the drop down list and click Delete (or drag in the trash icon at the bottom

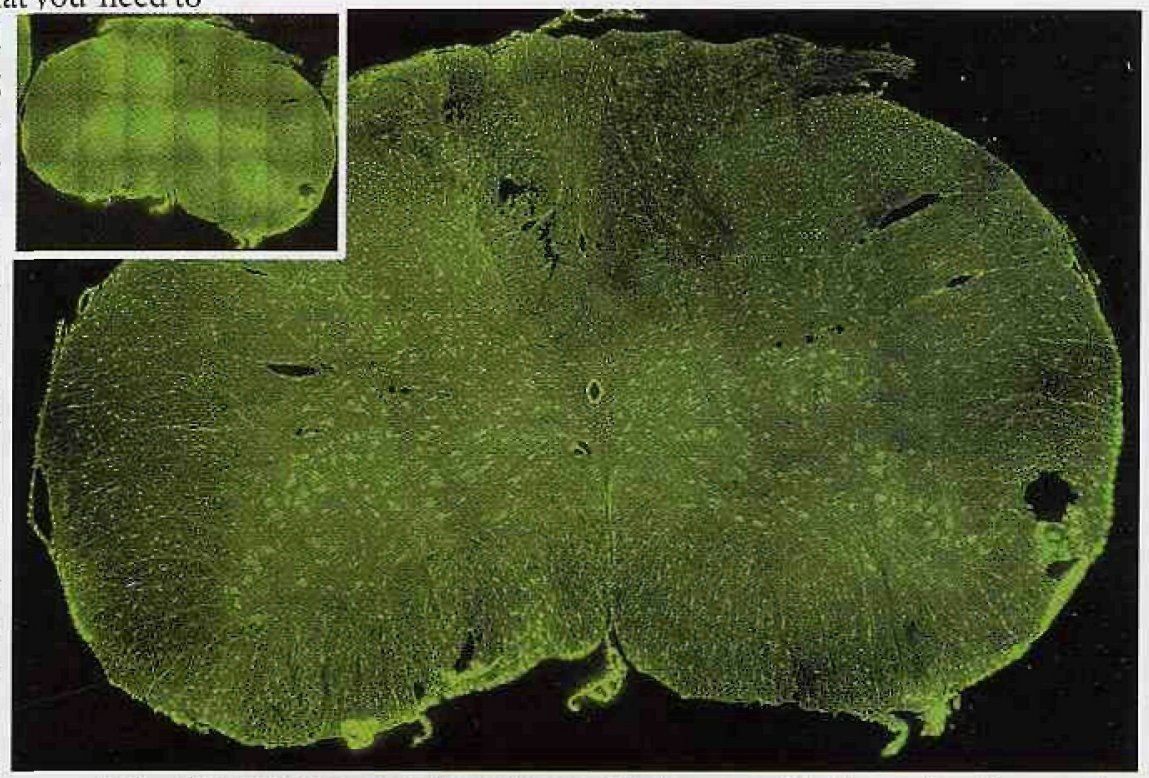

Fig5. After making rows, make another template to accomodate the number of rows and the width. You can record another action while moving rows into place. Inset shows what occurs when shading correction is not used with the camera (if available), or when images do not have enough overlap. 
of the Layers dialogue box). Be sure to click on the stop icon at the bottom of the Actions dialogue box after you do that.

Numeric Values.

You can move images by typing in $x$ and $y$ positions. This function is called "Numeric" in Photoshop $5 \mathrm{x}$, and in $6 \mathrm{x}$ and $7 \mathrm{x}$ it is available on the submenu. It is available when you choose Transform (Under Edit, choose Transform; also choose Numeric in $5 x$ ). After manually moving Layer 2 and Layer 3 into place, stop recording the action by clicking the stop button at the bottom of the Actions dialogue box. Click on the triangle at the left of the Move Current Layer step in the Actions dialogue box and look at the $x$ and $y$ values. You can see the amount you moved between the 2 nd and 3 rd layer, and by subtraction you can figure the difference. You can then determine where to place Layer 4, as long as your stage is very accurate. Click the Record button in the Actions dialogue box and continue. Under Edit, choose Transform (and then Numeric in 5x). Place the number you have determined into the $x$ and/or $y$ boxes (be sure Relative is checked in Photoshop $5 \mathrm{x}$ ). The layer will move automatically into place. Note that some stages move in a shorter distance when moving to the 2 nd image in a row than subsequent images, so you may want to move a 4 th layer manually into place in order to be certain of the $x, y$ travel in pixels.

\section{Save Completed Row.}

Be sure to save the row you've created into a separate folder. I call this folder "All Rows." If you are satisfied with the way the row looks, then flatten all the layers and save the image as a TIFF file (under Layer on the menu, select Flatten Layers). If you need to correct for subtle changes in brightness and contrast, or for uneven illumination issues, then leave the row in layers and save as a Photoshop image. It is best to adjust for these changes when the entire montage is assembled because a target exposure used for matching all other individual images is easier to identify when all images are on the same page.

\section{Combine Rows together to make final image.}

Repeat above steps for each row (make template manually, run Batch Automation using "Select All, Cut, Close, Paste," click on the arrow key at the bottom of the Actions dialogue box after clicking and highlighting "Stitch Into Rows"). Now make a final template into which rows can be cut and pasted after determining the final dimensions. Again, under File $>$ Automate $>$ Batch, run the "Select All, Cut, Close, Paste" command, only this time do so on the file folder named "All Rows." Again all the rows will be pasted on top of each other in the final template. Create a new action called "Combine Rows," and then move each layer into place starting with Layer 1. Be sure to stop the action when done.

If all goes well, images will match up with some overlap. The final image is completed, which can be saved at the large file size for full resolution as a photoshop file, and then saved as a second image as a TIFF file at a target resolution of 400 dots per inch at no more than 8.5 or so inches across (assuming a publication size of a full page on standard letter pages).

Assuming good protocol from the start (good alignment of camera, stage and microscope slide, shading correction, manual settings on the camera to avoid uneven brightness) images should be seamless or nearly seamless.

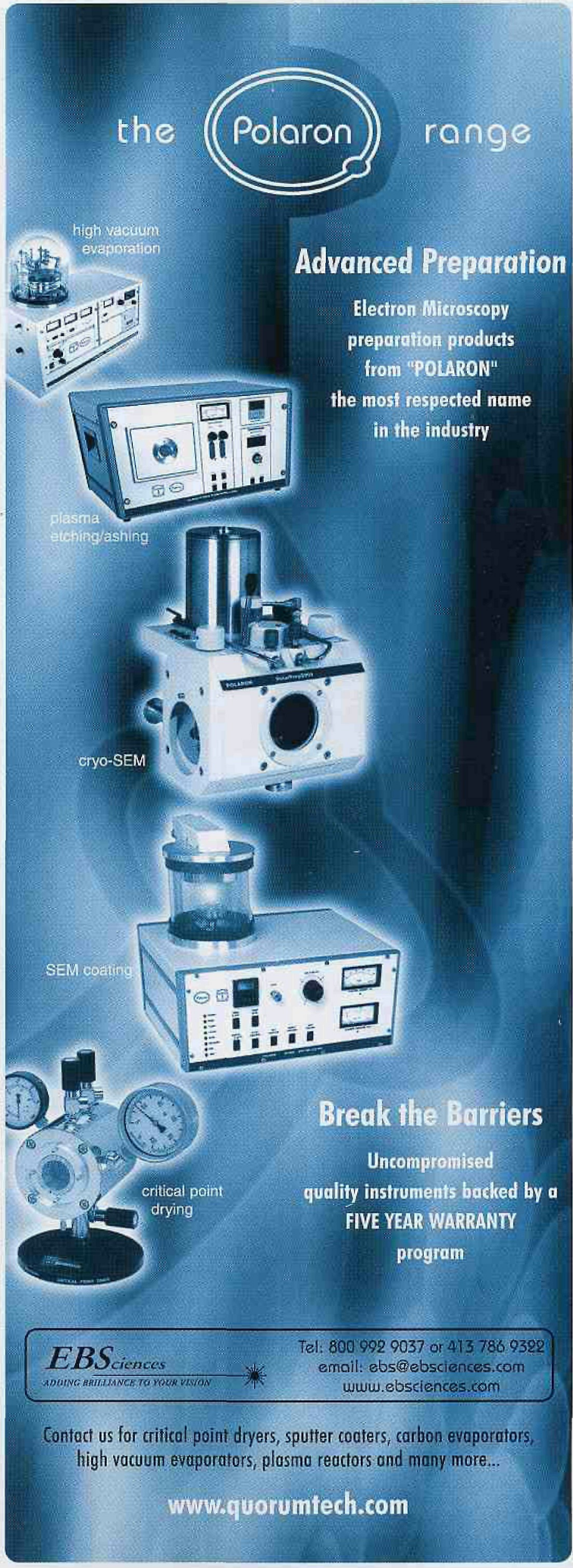

mIOROSOOPY TODAY January/February 2004 